\title{
L'imagerie romaine et sa contestation dans le discours révolutionnaire (1789-1807)
}

\section{Hélène Parent}

\section{(2) OpenEdition}

1 Journals

Édition électronique

URL : http://journals.openedition.org/rhetorique/1042

DOI : $10.4000 /$ rhetorique. 1042

ISSN : 2270-6909

Éditeur

UGA Éditions/Université Grenoble Alpes

Édition imprimée

ISBN : 978-2-37747-248-2

\section{Référence électronique}

Hélène Parent, «L'imagerie romaine et sa contestation dans le discours révolutionnaire (1789-1807) 》, Exercices de rhétorique [En ligne], 15 | 2020, mis en ligne le 07 novembre 2020, consulté le 03

décembre 2020. URL : http://journals.openedition.org/rhetorique/1042 ; DOI : https://doi.org/10.4000/ rhetorique. 1042

\section{Ce document a été généré automatiquement le 3 décembre 2020.}

\section{(c) (P)}

Les contenus de la revue Exercices de rhétorique sont mis à disposition selon les termes de la Licence Creative Commons Attribution - Pas d'Utilisation Commerciale - Partage dans les Mêmes Conditions 4.0 International. 


\title{
L'imagerie romaine et sa contestation dans le discours révolutionnaire (1789-1807)
}

\author{
Hélène Parent
}

1 À la veille de la Révolution française, Louis-Sébastien Mercier confie certains de ses regrets dans son Tableau de Paris. Il déplore entre autres l'absence d'une «tribune aux harangues, où l'on parleroit au public assemblé » :

On y tonneroit contre de cruels abus, qui ne cessent en tous pays, que quand on les a dénoncés à l'animadversion publique. Les plus beaux morceaux d'éloquence qui nous restent de l'antiquité, sont émanés de la tribune; \& aujourd'hui que les lumières politiques deviennent plus saines, on y proposeroit ce qui peut être utile au public ${ }^{1}$.

Le Tableau de Paris est publié entre 1781 et 1788 . Un an plus tard éclate la Révolution, et voici le vœu de Mercier exaucé : la France se voit dotée d'une assemblée parlementaire dont les débats sont publics et qui, notamment dans les périodes de crises politiques majeures, devient cette fameuse « tribune aux harangues » où l'on « tonne » volontiers. Cette situation se poursuit jusqu'en 1807, date à laquelle l'empereur Napoléon supprime le Tribunat, dernière assemblée délibérante dans les institutions impériales, mais dont les voix étaient déjà bien étouffées depuis la fin du Consulat. Entre 1789 et 1807, on assiste donc à la renaissance brutale du genre délibératif et de la grande éloquence politique, ayant pour but, depuis l'Antiquité, de décider de ce qui est utile ou nuisible pour la cité, donc pour le bien commun. Cette renaissance imprévue s'accompagne d'un certain nombre de débats et de querelles sur l'éloquence: quel langage convient-il de parler à cette tribune naissante ? Certaines questions, nouvelles, se posent: est-il possible de se référer à un modèle rhétorique dépassé, associé à l'Ancien Régime et aux institutions religieuses, quand on prétend faire table rase de ce passé et régénérer la société ? La grande éloquence déclamatoire ne s'oppose-t-elle pas à l'action politique, et donc à l'efficacité ? D'autres questions, issues d'une tradition ancienne, continuent de se poser: les ressources de la rhétorique, et notamment les ornements du discours, en faisant appel aux passions de l'auditoire, ne sont-ils pas 
trompeurs? Est-il moralement acceptable de solliciter l'imagination du peuple par le biais d'un langage figuré plutôt que de s'en tenir à la raison, alors même que cette dernière est la valeur qui domine dans le discours hérité des Lumières dont les révolutionnaires revendiquent la paternité ?

2 Qui s'adonne à la lecture des discours d'assemblées de la Révolution française est frappé par ce constat : il n'existe à première vue aucune réponse claire à ces questions, et toutes sont marquées du sceau d'une contradiction qui peut légitimement apparaître comme une terrible mauvaise foi de la part de bien des orateurs. En effet, tout en dénonçant, pour des raisons esthétiques mais surtout morales, l'usage d'une rhétorique classique inspirée en particulier de Cicéron et notamment des ressources de l'élocution, la grande majorité des orateurs a recours à tous les artifices de cette même rhétorique, et non des moindres, puisque l'éloquence révolutionnaire nous est généralement (et tristement) connue à travers ses " prosopopées de rhéteur ${ }^{2}$ » et autres formules " d'un faux goût d'Antiquité [décorant] les discours de toutes sortes d'ornements mythologiques grecs, romains ${ }^{3}$ ». Ces «ornements» que déplore Gustave Lanson, consistent précisément en des figures de rhétorique, la plus répandue dans le corpus de discours retenu étant la figure d'analogie construite à partir de la référence antique, parfois grecque, mais le plus souvent romaine. Tant dans le contenu - les références explicites à des épisodes ou à des noms propres tirés de l'histoire romaine - que dans la forme - l'imitation des tours cicéroniens, par exemple-, c'est donc surtout l'imprégnation rhétorique des discours que l'on retient de l'éloquence révolutionnaire. Mais alors pourquoi, quand bien même ce modèle latin et romain fait l'objet d'un rejet depuis la seconde moitié du xvIII siècle, les orateurs révolutionnaires y ont-ils si souvent recours? Comment expliquer la présence si massive de l'imagerie romaine dans leurs discours?

3 Les orateurs de la Révolution française revendiquent une continuité (certes construite a posteriori) entre leur œuvre et la pensée des Lumières. Dans la lignée des philosophes $\mathrm{du} \mathrm{xVIII}^{\mathrm{e}}$ siècle, ils valorisent la raison et dénoncent ce qui relève des passions, lesquelles sont liées aux préjugés qu'ils tâchent justement de détruire. Le procès intenté aux figures, et plus particulièrement aux images, est donc avant tout un procès à l'imagination, faculté sur laquelle prennent appui les passions. Pourtant, la cohabitation presque systématique, dans les discours, de cette accusation et d'un usage massif des figures, et notamment des figures d'analogie puisant dans la mémoire des références romaines, laisse songeur: cette dénonciation ne s'apparenterait-elle pas, somme toute, à une forme de lieu commun? En réalité, tout au long de la période révolutionnaire, l'éloquence délibérative éprouve un réel besoin des figures, surtout en un temps d'intense crise politique, où les luttes la font glisser vers le genre judiciaire, et où la figure construite à partir de l'imaginaire romain devient une arme indispensable du registre polémique.

\section{Du danger des « ornements » du discours : le procès de l'imagination}

\section{De l'imagerie romaine, et de son usage figuré}

4 Nous proposons d'appeler, de façon très générale, "imagerie romaine » toutes les formes textuelles qui posent ou supposent une analogie entre le passé romain et le 
présent révolutionnaire. Elles apparaissent dans différentes configurations au sein desquelles intervient, dans la plupart des cas, le discours figuré. Nous pouvons en appréhender la diversité sur un continuum allant du lieu commun littéral à la figure de pensée :

- L'exemple: à partir d'un cas particulier tiré de l'histoire romaine, l'auditoire est invité à tirer des conclusions actuelles.

- L'exemplum : à partir d'un cas particulier tiré de l'histoire romaine, l'auditoire est invité à tirer des conclusions à portée morale et universelle ${ }^{4}$.

- Le parallèle: une situation ou un personnage romains sont présentés, et mis en parallèle avec la situation présente, et il revient à l'auditeur d'opérer la comparaison entre ces deux situations ${ }^{5}$.

- La comparaison : le parallèle est alors explicité grâce à un outil de comparaison.

- La métaphore : le comparant romain se substitue totalement au comparé, et l'image romaine ne fait plus qu'une avec la réalité présente (on peut également classer dans cette catégorie certaines hypotyposes, en tant que métaphores en mouvement, souvent imitées des auteurs latins).

- La prosopopée : cette figure est fondée sur la substitution de la voix du personnage ou de l'allégorie romaines (donc d'un personnage mort ou fictif) à celle de l'orateur ${ }^{6}$.

Prégnante dans notre corpus, cette imagerie romaine, que l'on trouve parfois sous une forme actualisée, parfois sous la forme d'images hypothétiques, circule d'un discours à l'autre, et participe de ce que l'on pourrait appeler, avec Paul Ricœur, une " redescription métaphorique ${ }^{7}$ », ou plutôt, en l'occurrence, une redescription imagée et romanisée, des événements révolutionnaires. Les orateurs, en s'appuyant sur ces représentations culturelles bien connues de la population instruite et qui se diffusent peu à peu dans toutes les strates de la société urbaine grâce à l'abondante production d'images et de journaux, cherchent tout autant à persuader leur auditoire qu'à souder une communauté nationale à travers la récupération et la transformation d'un imaginaire commun. Les expressions imagées construites à partir du matériau romain valent donc, à ce double niveau, pour leur efficacité.

\section{Les expressions imagées : utiles, mais dangereuses}

6 Une longue tradition rhétorique a mis en évidence l'efficacité des images (allant de pair avec leur séduction éventuellement dangereuse), en insistant sur leur impact sensible. Chez Quintilien par exemple, le bon orateur est celui qui parvient à toucher, non seulement l'oreille, mais encore l'œil de son auditoire :

L'ornement est quelque chose de plus que ce qui est seulement clair et plausible. [...]

C'est une grande qualité que de présenter les choses dont nous parlons avec une

telle clarté qu'elles semblent être sous nos yeux. Le discours, en effet, ne produit pas un effet suffisant et n'exerce pas pleinement l'emprise qu'il doit exercer, si son pouvoir se limite aux oreilles et si le juge croit qu'on lui fait simplement le récit des faits qu'il connaît, au lieu de les mettre en relief et de les rendre plus sensibles au regard de son intelligence ${ }^{8}$.

Ce «regard de l'intelligence " renvoie à l'imagination, soit la faculté de créer des images mentales, et ce sont bien les figures, et plus précisément les tropes ${ }^{9}$, qui permettent de solliciter cette faculté en mettant « sous les yeux » de l'auditeur ce que l'on veut véritablement lui montrer. Or ces « ornements », de rigueur dans la rhétorique épidictique, prennent une dimension bien différente dans les discours judiciaire ou 
délibératif, ces derniers visant à agir sur le réel. En leur sein, l'expression figurée présente le risque de substituer au discours vrai une représentation relevant du mensonge. Le discours d'assemblée révolutionnaire se trouve inévitablement pris dans cette tension entre les préceptes de la rhétorique classique - à laquelle les orateurs de la Révolution française ont été formés - et le problème moral posé spécifiquement par la parole politique. Le cas de Saint-Just en offre un bon exemple. Ce dernier cultive une haine farouche pour la rhétorique: son idéal, d'inspiration fortement rousseauiste, réside dans un laconisme spartiate, dans la recherche d'un langage épuré et parfaitement transparent. Le plan d'éducation qu'il présente dans ses Institutions républicaines, un texte théorique rédigé au cours des années 1793 et 1794, rejette violemment la rhétorique et ses ressources :

Les enfants sont rigoureusement formés au laconisme de langage: on doit leur interdire les jeux où ils déclament et les accoutumer à la vérité simple. [...].

Les lycées distribueront des prix d'éloquence.

Le concours pour les prix d'éloquence n'aura jamais lieu par des discours d'appareil. Le prix de l'éloquence sera donné au laconisme, à celui qui aura proféré une parole sublime dans un péril [...].

Les enfants seront élevés dans l'amour du silence et du laconisme et dans le mépris des rhéteurs ${ }^{10}$.

7 À rebours de tout ce que Saint-Just a lui-même appris au collège oratorien de Soissons, son plan d'éducation oppose l'éloquence, qu'il définit comme un art de la brièveté sublime, à la technique des «rhéteurs ». La rhétorique des " discours d'appareil » est située aux antipodes de la "vérité simple", associée par l'orateur à un style sans ornement. Pourtant, le 9 thermidor an II, le jour du coup d'État qui provoque la chute de Robespierre et de ses alliés, le même Saint-Just, tentant de prendre la défense de «l'Incorruptible » - qui se présente volontiers comme le « consul de Rome » quand ses détracteurs le surnomment le «tyran de l'opinion»- monte à la tribune pour prononcer ce qui s'apparente, selon Anne Quennedey, à un "vibrant éloge de l'éloquence ${ }^{11} »$. Et, ce faisant, il commence par poser une question d'ordre purement rhétorique : "[q]uel langage vais-je vous parler? Comment vous peindre des erreurs dont vous n'avez aucune idée, et comment rendre sensible le mal qu'un mot décèle, qu'un mot corrige ${ }^{12}$ ?» Cette interrogation peut évidemment être lue comme une question oratoire dénonçant la surdité des députés, mais on peut aussi y voir le questionnement philosophique d'un théoricien de la bonne éloquence (un rhéteur, en somme) se demandant quel est le langage adapté à l'éloquence délibérative en temps de crise politique. Or, la réponse se trouve dans les verbes "peindre» et "rendre sensible », qui reviennent finalement à l'injonction cicéronienne de recourir à une éloquence visuelle et donc à des expressions figurées, surtout lorsque l'urgence de la situation ne permet plus de s'en tenir au laconisme, à « un mot » frappant l'oreille et qui, ordinairement, pourrait suffire. Saint-Just, dans le chaos du 9 thermidor, se heurte de plein fouet à la contradiction qui sous-tend tout discours sur les figures: dangereuses, moralement condamnables, elles sont également indispensables à l'efficacité du discours, sans laquelle l'éloquence politique n'a guère de sens.

\section{Garat et Lequinio à la chasse aux figures}

8 Pourtant, cette condamnation des figures et de leur corollaire, l'imagination, est récurrente dans des textes théoriques produits au cours de la période révolutionnaire, souvent par des témoins qui, prétendant juger «à froid» les déclamations de leurs 
collègues, écrivent de véritables réquisitoires contre l'éloquence des assemblées. C'est notamment le cas de Garat et Lequinio, deux exemples intéressants car ils nous montrent bien que cette condamnation des ressources de la rhétorique est universelle, et non pas liée à une idéologie ou à un camp politique en particulier.

Garat est un député de l'assemblée constituante, proche des constitutionnels ${ }^{13}$. Devenu ministre au cours des années 1792-1793, il est accusé de complicité avec la Gironde suite aux journées des 31 mai et 2 juin $1793^{14}$. Au cours de l'an III, il rédige un Mémoire sur la Révolution, où il revient sur ces accusations et commente les luttes oratoires qui animaient alors la Convention. Il insiste sur le danger des figures d'analogie romaines, et sur leur caractère démagogique. Par exemple, lorsqu'est nommée le 21 mai 1793 la commission des Douze ${ }^{15}$, il note que ses membres sont rapidement surnommés par les montagnards les « décemvirs » et qu' " avec ce mot, qui souleva, il y a trois mille ans, le peuple de Rome, on soulevoit la multitude de Paris ${ }^{16}$ ». Aux girondins, Garat reproche de faire un usage dévoyé de l'imagerie romaine et de l'imitation rhétorique: «[j]e trouve très bon qu'on tâche d'imiter les mouvemens passionnés de l'éloquence de Cicéron; mais je voudrois aussi qu'on imitât l'habileté de sa conduite ${ }^{17}$ ». Par cette suggestion, Garat valorise l'imitation à partir du moment où elle concerne en priorité une attitude politique (l'habileté d'un Cicéron privilégiant la raison d'État), et non un style rhétorique. Dans le même ordre d'idée, il affirme, à propos de Robespierre, que « jamais homme sachant écrire des phrases intelligentes et belles ne fut plus étranger à la bonne logique ${ }^{18}$ ", ou encore à propos de Guadet, qu'il l'a « conjuré [...] de renoncer, quelques fois au moins, aux triomphes de cette éloquence qu'on puise dans les passions, mais qui les nourrit et les enflamme ${ }^{19}$ ".

Lequinio, quant à lui, est un conventionnel montagnard plutôt discret à l'assemblée. Le 9 novembre 1792, il publie un ouvrage intitulé Les préjugés détruits, dont le chapitre VII s'intitule «De l'éloquence ». Il commence par y définir brutalement l'éloquence comme "l'art de tromper les hommes en leur faisant aimer leur erreur ${ }^{20}$ ", puis il oppose à la pratique de l'orateur celle du philosophe. Or, qu'est-ce qui diffère, entre le style du philosophe et celui de l'orateur? Justement, l'usage des "ornements» et "parures oratoires ", dont relèvent en particulier les figures :

La raison est simple et n'exige aucun ornement pour avoir tout son prix : celui qui chercher à l'habiller de parures oratoires, a besoin de vous tromper, il faut qu'il vous séduise et qu'il vous entraîne, c'est là son but ${ }^{21}$.

\section{L'imagination et les figures au tribunal des orateurs}

11 Mais ceux qui écrivent des textes théoriques en se présentant comme de simples observateurs, tels Garat et Lequinio, ne sont pas les seuls à dénoncer les ravages des figures et de l'imagination qu'elles mettent en branle : les orateurs, au sein de leurs discours eux-mêmes, en font état. De fait, si l'on observe plusieurs exemples de discours, on remarque que le terme "imagination", quel que soit l'orateur qui le prononce, est toujours employé dans un sens péjoratif, et par opposition à la vérité des «faits ». Le 21 juillet 1792, le constitutionnel Dumolard s'attaque à l'« imagination barbare » des "folliculaires ${ }^{22}$ " jacobins. Début novembre 1792, le girondin Pétion reproche à Robespierre ses «déclamations animées" provoquées par une " imagination sombre ${ }^{23}$ » ou encore " atrabilaire $^{24}$ ", quand lui-même revendique «le langage austère de $\mathrm{la} \operatorname{loi}^{25}$ ». Le 12 avril 1793 , Guadet se défend de sa prétendue complicité avec le traître Dumouriez, général proche des girondins et passé à l'ennemi 
peu de temps auparavant: "[e]n deux mots, je n'ai jamais eu de liaison avec Dumouriez; ce ne sont pas là des imaginations, ce sont des faits ${ }^{26}$ ». Et, le $1^{\text {er }}$ août 1793 , c'est au tour de Barère de dénoncer les complices du ministre anglais Pitt, lequel aurait tâché d'encourager les fédéralistes lyonnais en leur envoyant de l'or: «Hommes du Midi, vous que la nature jeta dans le moule brûlant des républicains, serez-vous donc toujours les victimes des intrigants qui parlent à votre imagination pour altérer vos principes $^{27}$ ? » Robespierre lui-même, accusé le 9 thermidor de s'être fait le «tyran de l'opinion " grâce à une éloquence démagogique, dénonce la veille de son arrestation la "légèreté académique " du style de ses adversaires, ainsi que leurs "phrases de rhéteurs ${ }^{28}$ ", et pointe du doigt leur usage vain et inconstant de la figure d'analogie romaine: "il y a trois jours, ils étaient prêts à me dénoncer comme un Catilina ; aujourd'hui ils me prêtent les vertus de Caton ${ }^{29} »$.

Ce rejet d'une imagination stimulée par des «déclamations » et des figures, qui ici n'intervient plus dans des textes théoriques, mais bien dans les discours des orateurs eux-mêmes, a de quoi surprendre : les orateurs qui prononcent ces discours ne sont-ils pas les mêmes à qui, par exemple, Garat reprochait d'exciter les passions en s'adonnant à la rhétorique cicéronienne et aux images romaines?

\section{Dénoncer l'usage des figures : un nouveau lieu commun?}

\section{Des figures qui s'en vont et qui reviennent}

13 En fait, on retrouve presque systématiquement, dans un discours dénonçant l'usage des ressources de la rhétorique et tout particulièrement les analogies avec l'imagerie romaine, un recours manifeste à ces mêmes figures. Pour le constater, il suffit de reprendre quelques-uns des discours précédemment mentionnés. Le 21 juillet 1792, lors du débat sur l'affaire La Fayette, qui oppose le camp constitutionnel au camp jacobin ${ }^{30}$, alors que Dumolard vilipende l'« imagination » jacobine et raille l'usage que font ses ennemis de la comparaison entre La Fayette et César, il n'hésite pas à comparer à son tour La Fayette au dictateur Camille, image entourée de connotations plus positives :

Parlez, Romains : bannirez-vous Camille lorsque les Gaulois sont à vos portes ! Représentants d'une grande nation, c'est plutôt sur ses destins que sur le sort de la Fayette que vous allez prononcer; et ce jour peut décider de votre gloire bien plus que de la sienne ${ }^{31}$.

Ici, le parallèle se présente sous la forme d'une image hypothétique : la substitution des " Romains » aux députés et de "Camille » à La Fayette est énoncée au futur mais, par le biais de cette figure, quoique non actualisée, la scène réelle glisse imperceptiblement vers un imaginaire romain bien connu du public cultivé. Le 12 avril 1793, alors que la lutte qui oppose les girondins aux montagnards bat son plein, Guadet, prétendant opposer des «faits " à l'« imagination " montagnarde, ouvre son discours en réfutant une analogie entre Robespierre et Cicéron, et entre lui-même et Catilina :

Citoyens, si en dénonçant devant le sénat de Rome celui qui avait conspiré contre la liberté de son pays ; si en dénonçant Catilina, Cicéron avait fondé son accusation sur des preuves de la nature de celles que Robespierre a produites contre moi, Cicéron n'eût inspiré dans l'âme de ceux qui l'eussent entendu que de l'indignation et du mépris. Mais si, après avoir annoncé qu'il venait de remplir un ministère douloureux et pénible, qu'il y était forcé par l'amour de sa patrie, Cicéron eût terminé son discours par une ironie ou une plaisanterie, Cicéron eût été 
honteusement chassé du sénat ; car, chez ce peuple, on détestait la calomnie, et on savait punir les calomniateurs. Mais Cicéron était un homme de bien, il n'accusait pas sans preuves. Cicéron n'eût pas spéculé sur l'ignorance du peuple; Cicéron n'aurait pas accaparé une réputation populaire, pour accaparer la république... Je m'arrête... Aussi bien que peut-il y avoir de commun entre Cicéron et Robespierre? Entre Catilina et moi $^{32}$ ?

Guadet, tout en refusant, grâce à un raisonnement basé sur le lieu commun de l'impossible, la double figure d'analogie utilisée par son adversaire, rebondit dessus et la développe dans un système hypothétique, sur le mode de l'histoire contrefactuelle, ce qui lui permet à la fois de démontrer l'emploi non pertinent de la figure romaine, et de la réutiliser pour son propre compte. Enfin, Robespierre lui-même, dans son discours du 8 thermidor an II, après avoir raillé les comparaisons à Caton et à Catilina relevées dans les discours de ses adversaires, affirme qu' "[il] [se] réjoui[t] de voir la fureur des Verrès et des Catilina de [son] pays tracer une ligne profonde de démarcation entre eux et tous les gens de bien ${ }^{33}$ ».

\section{Une double stratégie rhétorique}

En réalité, il semble donc bien que le rejet des figures ne soit finalement qu'un soutien à l'efficacité des figures elles-mêmes, à la manière d'un nouveau lieu commun qui fonctionnerait sur le mode de la prétérition: en prétendant ne pas dire, et en dénonçant ce que l'adversaire dit, on s'octroie la possibilité de dire soi-même, voire d'en dire davantage. Le refus de la figure au nom de la raison relève donc d'une stratégie double, permettant à la fois de disqualifier l'adversaire et de justifier son propre usage des figures, tout en renforçant leur effet.

Ainsi, le 29 octobre 1792, le girondin Louvet, accusant Robespierre d'aspirer à la dictature, se lance d'abord dans une énumération d'exemples (ou d'exempla, dans la mesure où ils font aussi office de repoussoirs moraux):

Ruse grossière, mais qu'on ne doit pas, quelque méprisable qu'elle soit, repousser par le seul mépris ; car on sait trop que c'est elle qui a réussi à tous les usurpateurs, à tous, depuis César jusqu'à Cromwell, depuis Sylla jusqu'à Masaniel ${ }^{34}$.

À ce stade, ce n'est que la « ruse » de Robespierre qui est pointée du doigt pour avoir aussi été celle des quatre dictateurs cités : il n'y a pas encore véritablement de figure. Mais Louvet sème ensuite quelques indices d'une autre analogie discrète, en qualifiant d'abord son adversaire d' $\mathrm{d}^{\text {' usurpateur superbe }}{ }^{35}$ ", l'adjectif faisant écho à Tarquin, puis d'« orgueilleux Robespierre ${ }^{36}$ ». Pour autant, il ne s'agit encore que d'une allusion vague. En revanche, ces deux réseaux d'images en préparation, l'un autour de César et Sylla, l'autre autour de Tarquin, finissent par se rejoindre dans une amplification au sujet des massacres de septembre :

De farouches conjurés venaient de cimenter par le sang leur autorité naissante ; et pour l'affermir, il leur fallait encore 28,000 cadavres! Alors je me ressouvins de Sylla, qui commença par frapper dans Rome des citoyens détestés, et qui bientôt fit porter sur les places publiques et sur la tribune aux harangues, les têtes des citoyens les plus recommandables par leurs vertus et leurs talents. Ainsi la faction désorganisatrice, escortée de la terreur et toujours précédée des placards de l'homme de sang, s'avançait rapidement vers son but ; ainsi les conjurés allaient sur les débris de toutes les autorités et de toutes les réputations, commencer leur règne ; ainsi tu marchais à grands pas, Robespierre, vers ce pouvoir dictatorial dont la soif te dévorait, mais où t'attendaient enfin plusieurs hommes de quelque résolution, et que, n'en doute pas, ils l'avaient juré par Brutus, tu n'aurais pas gardé plus d'un jour. (Applaudissements réitérés ${ }^{37}$.) 
Le parallèle entre la Montagne et Sylla se développe d'abord à travers une comparaison ("Ainsi la faction désorganisatrice [...] ») mais, peu à peu, la figure du seul Robespierre se superpose à celle de Sylla, au sein d'une apostrophe ("tu marchais à grands pas, Robespierre »), et la mention du « règne » fait fusionner l'image du dictateur et celle du roi. Ces deux images se trouvent d'ailleurs réunies dans le nom «Brutus» qui peut désigner simultanément deux personnages, le fondateur de la république et l'assassin de César, puisque le terme " juré » fait écho au serment du premier, et que la punition rapide du dictateur évoque plutôt le geste tyrannicide du second. Encore Louvet ne compare-t-il pas directement son camp à Brutus, préférant faire de ce dernier le simple témoin du serment girondin. Fausse modestie ou méfiance vis-à-vis des figures d'analogie trop faciles, qu'importe : ce qui compte, c'est que les connotations soulevées par le nom propre «Brutus » et préparées par le reste du discours suffisent à ce que l'auditoire assimile en fait les girondins au héros romain, et les «applaudissements réitérés » témoignent de l'efficacité des figures et de l'amplification oratoire.

Le 5 novembre 1792, Robespierre répond à son accusateur. Il commence par dénoncer son style: "[a]ux déclamations les plus absurdes et les plus atroces, comme aux suppositions les plus romanesques et les plus démenties hautement par la notoriété publique, je ne réponds que par les faits ${ }^{38} »$. Mais, contrairement à ce qu'il affirme, Robespierre ne s'en tient pas aux « faits », puisque quelques lignes plus loin, il reprend et déplace une analogie avec Catilina, dressée précédemment par Barbaroux, un autre orateur girondin :

Quand le consul de Rome eut étouffé la conspiration de Catilina, Clodius l'accusa d'avoir violé les lois. Quand le consul rendit compte au peuple de son administration, il jura qu'il avait sauvé la patrie, et le peuple applaudit. J'ai vu, à cette barre, des citoyens qui ne sont pas des Clodius, mais qui, quelques temps avant la révolution du 10 août, avaient eu la prudence de se réfugier à Rouen, dénoncer emphatiquement la conduite du conseil de la Commune de Paris ${ }^{39}$.

Robespierre ne mentionne pas le nom de Cicéron, il préfère user de deux périphrases et évoquer, de manière très allusive, deux épisodes bien connus de la vie du « consul de Rome ». Ensuite, Robespierre nie, à travers une concession, le parallèle qu'il vient d'établir entre Clodius et les girondins ("J'ai vu, à cette barre, des citoyens qui ne sont pas des Clodius, mais [...]»). La concession, ici, permet à la fois de prévenir le reproche habituel fait à l'encontre des figures et, comme la prétérition, de glisser la figure d'analogie tout en prétendant la réfuter, pour finalement la faire ressortir. De cette façon, il réussit le tour de force, non seulement d'endosser implicitement l'ethos de Cicéron (donc de retourner l'imaginaire négatif de Catilina en son contraire), mais encore de retourner l'accusation qu'on lui fait d'être un Catilina en lui faisant subir un déplacement : les girondins ne sont pas des Catilina mais des Clodius (encore ne le sontils pas au sens strict), ce qui n'est guère plus reluisant.

\section{Thermidor : la fin des figures?}

17 Mais la dénonciation des figures, si elle traverse toute la période révolutionnaire, est surtout restée dans les esprits comme un lieu commun du discours thermidorien, à l'encontre du discours jacobin. Qu'en est-il de cette croyance? L'imagerie romaine, accusée d'avoir, en stimulant l'imagination et les passions, suscité la Terreur, tombe-telle vraiment aux oubliettes après Thermidor? 
18 Nombreux sont les textes théoriques qui voudraient nous le laisser penser, en assimilant le recours aux figures à la rhétorique jacobine. Ainsi, l'idéologue Volney, dans son cours à l'école normale de l'an III, affirme que ce sont

ces livres classiques si vantés, ces poètes, ces orateurs, ces historiens qui [...] offrant [à la jeunesse] pour modèles certains hommes, certaines actions, l'ont enflammée du désir si naturel de l'imitation; qui l'ont habituée sous la férule collégiale à se passionner pour des vertus et des beautés réelles ou supposées, mais qui, étant également au-dessus de sa conception, n'ont servi qu'à l'affecter du sentiment aveugle appelé enthousiasme ${ }^{40}$.

C'est donc l'imitation, aussi bien des héros que des rhéteurs de l'Antiquité - deux modalités que Volney ne distingue pas, confondant ainsi l'ordre du réel et l'ordre du discours -, qui serait à l'origine de l'imagerie romaine et qui aurait soulevé les passions menant à un "enthousiasme " dévastateur. De la même manière, La Harpe, dans le discours qu'il prononce à l'ouverture du Lycée le 31 décembre 1794 et qui fait office d'introduction à ses Cours de littérature ancienne et moderne, qualifie encore bien plus violemment l'éloquence jacobine de «mélange inouï de dépravation monstrueuse et de rhétorique puérile, de jactance emphatique et de grossièreté triviale ${ }^{41}$ ». Il accuse le «charlatanisme de tréteaux» des orateurs, qu'il associe précisément à l'usage des figures d'analogie, et donc à l'abus des mots: «[p]roclamant des milliers de meurtres au nom de l'humanité, le code du brigandage au nom d'Aristide; consacrant la plus exécrable tyrannie au nom de Brutus ${ }^{42}$ ». Enfin, après avoir ainsi accusé les jacobins de dévoyer le sens des mots en l'inversant par le biais d'analogies mensongères, il énonce son propre idéal sous la forme d'une expression lapidaire relevant elle-même quasiment d'un topos rhétorique : «[p]arlons sans figure ${ }^{43} »$.

«Sans figure ", le discours thermidorien ? Pourtant, Courtois, en janvier 1795, dans son Rapport au nom de la commission chargée de l'examen des papiers trouvés chez Robespierre et ses complices prononcé à la Convention en janvier $1795^{44}$, recourt massivement à l'imagerie romaine : cette présence est notable, dans la mesure où le texte en question aura un rôle important dans la construction de la légende noire de Robespierre. Et de même, Dussault, dans son pamphlet intitulé Véritable portrait de Catilina-Robespierre, tiré d'après-nature, forge un nouveau nom propre ( «Catilina-Robespierre $\left.{ }^{45} »\right)$ inspiré d'un discours de Fréron prononcé le jour même du 9 thermidor : or ce texte connaît un succès remarquable au cours de l'an III... Une fois de plus, le lieu commun consistant à dénoncer l'usage des figures n'est, dans le discours thermidorien, qu'une stratégie rhétorique qu'on pourrait presque qualifier de figure à son tour, à la nuance près que ce discours est sorti durablement vainqueur de la bataille rhétorique ${ }^{46}$. Au reste, les querelles entre les différentes factions révolutionnaires doivent être lues comme des querelles d'ethos: en s'attaquant à l'imagerie romaine, sous toutes ses formes, c'est en fait l'ethos de ses adversaires que déconstruit le discours thermidorien. Mais, quoiqu'il prétende rejeter ces figures, il ne peut déconstruire l'ethos jacobin qu'en le renversant, et en le reprenant à son compte, comme on peut le voir dans le discours de Courtois, où Robespierre, qui se qualifiait pourtant lui-même de "consul de Rome », devient un avatar de Néron ou de Catilina, tandis que l'image de Cicéron sauvant la patrie est mise au service de la construction de l'ethos thermidorien dans le cadre de la polémique. En somme, les références romaines, apparaissant sous forme de figures, constituent bien ce « terrain commun entre les entreparleurs ${ }^{47}$ » décrit par Marc Angenot au sujet de la parole polémique. 


\section{Figures de la Révolution, révolution des figures : les nouveaux enjeux de l'image romaine}

\section{Indispensables figures}

Ainsi les figures, bien que décriées, demeurent une arme dont les orateurs ne peuvent se passer et dont ils optimisent l'efficacité, tout particulièrement en période d'intense crise, dans le contexte de la lutte oratoire. On a vu comment Saint-Just, dans sa tentative pour prendre la défense de son mentor le 9 thermidor, s'interrogeait sur le langage à adopter dans une situation telle que la sienne ${ }^{48}$. Et, de fait, c'est bien grâce à une métaphore issue de l'imaginaire romain que ce même Saint-Just, lui qui affirme détester les ornements de la rhétorique, défend l'éloquence robespierriste. Ayant recours au lieu commun des contraires, et l'illustrant à travers deux exemples/exempla tirés de l'histoire romaine ou plus généralement antique (la vertu et l'éloquence de Caton l'Ancien, parangon du vir bonus dicendi peritus, versus le silence qui règne autour du trône de Philippe de Macédoine), il superpose à la figure de Robespierre celle de Caton, et aux figures de ses ennemis celles des courtisans de Philippe, créant ainsi une scénographie où la «tribune aux harangues » des Romains en vient à remplacer la tribune réelle de la Convention :

On [...] constitue [Robespierre] en tyran de l'opinion: il faut que je m'explique làdessus, et que je porte la flamme sur un sophisme qui tendrait à faire proscrire le mérite. [...] Êtes-vous donc de la cour de Philippe, vous qui faites la guerre à l'éloquence? Un tyran de l'opinion? Qui vous empêche de disputer l'estime de la patrie, vous qui trouvez mauvais qu'on la captive ? [...] Caton aurait chassé de Rome le mauvais citoyen qui eût appelé l'éloquence dans la tribune aux harangues, le tyran de l'opinion. [...] Ainsi, la médiocrité jalouse voudrait conduire le génie à l'échafaud! Eh bien, comme le talent d'orateur que vous exercez ici est un talent de tyrannie, on vous accusera bientôt comme des despotes de l'opinion ${ }^{49}$.

Saint-Just retourne habilement l'accusation : les « despotes de l'opinion ", eux-mêmes orateurs, ne valent pas mieux que le «tyran de l'opinion », et leurs reproches, selon lui, ne témoignent que d'une "médiocrité jalouse ». Ainsi, la figure, objet de rejet, se retourne subitement en objet d'émulation, et le refus des figures se transforme paradoxalement en une course aux figures: la lutte politique se double d'une lutte rhétorique, qui dans le cas précis vient presque s'y substituer.

D'ailleurs, dans le champ des rhétoriciens, on retrouve un paradoxe similaire. En effet, au cours de cette période de renaissance de l'éloquence délibérative, et alors même que tout un chacun -orateurs, journalistes, écrivains - semble avoir son mot à dire sur l'art de la parole et de la persuasion, il est remarquable qu'un seul traité de rhétorique en bonne et due forme soit publié. Or, il s'avère que ce traité de Joseph Droz, intitulé Essai sur l'art oratoire (1800), fait des figures une présentation plutôt positive. En ouvrant le chapitre IX de cet ouvrage, sous-titré « Observations sur les figures ${ }^{50}$ », l'auteur déplore qu'on soit "parvenu à rendre puéril et pédantesque une partie de l'art d'écrire, qui pourrait être intéressante ${ }^{51}$ ». Et si cette partie de la rhétorique est «intéressante », c'est justement parce que, bien loin d'être mensongère, elle est souvent l'unique truchement qui mène à la vérité : «[s]ouvent c'est à [la figure] seule que vous devez la vérité avec laquelle vous vous énonce $z^{52} »$. Dans la lignée d'Aristote et de Cicéron, Droz insiste à cet égard sur les figures d'analogie, comparaisons et métaphores, car elles permettent d'éclairer une « idée abstraite qui [...] est rendue sensible par une image ${ }^{53}$ ». 
Et il conclut ce chapitre consacré aux figures par un passage qui ne peut que faire écho à la situation dans laquelle se trouvent les orateurs de la période révolutionnaire :

La source, la véritable source du style figuré, est le besoin de peindre ses idées pour s'exprimer avec vérité. C'est dans l'enfance des sociétés qu'on en fait le plus fréquent usage, parce que les sensations qu'éprouvent des hommes neufs, sont plus vives que celles des hommes policés ${ }^{54}[. .$.$] .$

Certes, Droz ne fait pas directement référence à la Révolution, puisqu'il semble ensuite opposer à la civilisation une sorte d'état primitif de l'humanité s'inscrivant dans la tradition philosophique d'une réflexion sur l'état de nature, très vivace depuis le début $\mathrm{du}$ siècle. Pourtant, c'est bien comme une « enfance » que les révolutionnaires vivent et décrivent la Révolution, et eux-mêmes se pensent comme des "hommes neufs", régénérés. Par ailleurs, comment le caractère inouï des événements révolutionnaires et la rapidité de leur succession pourraient-ils passer outre la nécessité du recours à un langage imagé pour les décrire ? L'imaginaire romain, connu de tous les gens cultivés et que les orateurs révolutionnaires contribuent à diffuser, offre un matériau imagé accessible et immédiatement partageable, qui appréhende de manière sensible une actualité brûlante dont la réalité ne peut être saisie par le concept: on retrouve là l'idée, présente chez Paul Ricœur, d'une métaphore qui « dévoile » une vérité cachée, ou du moins, dans le cas précis, une réalité qu'on ne parvient pas à expliquer et qu'il est donc nécessaire de sentir par l'intermédiaire du langage figuré.

\section{Métamorphoses de la métaphore : un réseau de figures}

Et ce qui rend l'imagerie romaine particulièrement importante dans le discours révolutionnaire, c'est la façon dont elle circule et dont elle est reprise d'un discours à l'autre, construisant ainsi un vaste réseau imaginaire autour duquel se cristallisent les émotions collectives. Nous avons déjà pu voir comment, tout en rejetant les figures utilisées par leurs adversaires, les orateurs les récupéraient pour leur compte. Voyons à présent plus précisément comment ces figures circulent et se transforment, à travers l'exemple de l'analogie entre La Fayette et César, en juillet-août 1792.

Le 21 juillet, le constitutionnel Dumolard accuse les jacobins d'être des «hommes habiles dans l'art de tromper le peuple ${ }^{55}$ » et cette accusation s'appuie sur la mobilisation récurrente, par ses adversaires, de la comparaison de La Fayette à César aspirant à la dictature :

La Fayette se rend-il à Paris seul, et n'ayant d'autre cortège que sa gloire et sa vertu; aussitôt la calomnie s'agite; une voix infernale se fait entendre : Tremblez Romains, César a passé le Rubicon! Je le demande à ces hommes de mauvaise foi ; César laissa-t-il ses légions dans les Gaules ? Pénètre-t-il dans Rome, et paraît-il au sénat dans l'attitude d'un pétitionnaire ${ }^{56}$ ?

Le fonctionnement de l'émotion oratoire mise en branle par la figure est bien décrit par l'orateur: le "rapprochement injuste » permet d'« épouvanter », et donc d'« entraîner la multitude ${ }^{57}$ ", et puisqu'elle est mensongère, cette figure devient "calomnie » À travers la reprise en mention ironique du discours jacobin - signalée à l'écrit par les italiques et probablement sensible dans le ton de l'orateur - ainsi que des questions oratoires, Dumolard s'attache à démontrer le caractère fallacieux de l'analogie entre La Fayette et César, mettant ainsi en lumière la «mauvaise foi » de ses adversaires. Mais cette dernière accusation ne manque pas de sel lorsque l'on sait que, dans la suite de son discours, Dumolard, en remplaçant l'image de César par celle de Camille ${ }^{58}$, renverse l'imaginaire négatif associé à La Fayette en lui substituant un imaginaire 
positif. Une quinzaine de jours plus tard, le constitutionnel Vaublanc, prenant à son tour la défense de La Fayette, se retrouve lui aussi face à la contrainte du langage figuré : « [c]'est avec cette froide modération que j'aurais voulu parler de la conduite du général La Fayette ; mais, attaqué par la fureur et l'animosité, il doit être défendu avec chaleur $^{59}$ ». Grâce à une sorte d'anté-occupation ${ }^{60}$, Vaublanc justifie par avance la "chaleur" de son éloquence par la nécessité dans laquelle il se trouve de placer sa défense à la hauteur de l'attaque. Se prétendant outré par le langage de ses adversaires, il affirme, dans une prétérition :

Je ne répondrai pas à toutes les phrases de collège, répétées avec complaisance sur le général La Fayette, pour travestir sa démarche en un attentat punissable [...]. Ces expressions emphatiques, ces comparaisons odieuses sont indignes de la tribune de cette assemblée, et ne feront sur vous d'autre impression, que celle que produit une injustice révoltante ${ }^{61}$.

En fait, non content de répondre aux "phrases de collège » et aux " déclamations ", Vaublanc les met lui-même en pratique, d'abord sous la forme d'une comparaison hypothétique, concernant La Fayette : « [j] dis que s'il avait eu des projets ambitieux et criminels, il n'aurait songé d'abord, comme César, Sylla, Cromwell, à fonder sa puissance sur des victoires avant de se déclarer ouvertement ${ }^{62}[. .$.$] »; puis sous la forme$ d'une métaphore, dirigée à l'encontre des jacobins : «[c]eux-là ne sont pas faits pour défendre la liberté, mais pour être assis à la cour des Tibère et de Néron ${ }^{63}$ ». Voilà un doublé de figures (métaphore et antonomase) qui ne ressemble effectivement pas à une "froide modération », l'opposition entre chaleur et froideur renvoyant ici, de façon imagée, à l'opposition entre la passion et la raison (et donc entre le movere et le docere).

Enfin, le même jour, le jacobin Brissot répond à Vaublanc et à Dumolard. Sous la forme d'une concession à demi-mot, il prétend être d'accord avec eux quant à l'absurdité de la comparaison entre César et La Fayette: «[j]e ne m'arrêterai point à son indignation que tout le monde partage, sur le parallèle entre César et M. La Fayette ${ }^{64} »$. Mais on comprend bien vite que ce qui gêne Brissot n'est pas tant l'usage de la figure que le choix du comparant, « car enfin, César n'a pas à citer en sa faveur qu'une égratignure et qu'une retraite savante ${ }^{65}$ ». Ainsi, tout comme Dumolard qui «rougirai[ $\left.t\right]$ aussi de mettre en parallèle La Fayette et César : César asservit sa patrie gémissante, et le nom de La Fayette est inscrit aux fastes de mémoire parmi le libérateur des deux mondes ${ }^{66} "$, Brissot dénonce l'inadéquation de l'image à la réalité, mais dans un sens inverse à celui de Dumolard. Contrairement à celui-ci, qui recourt à l'imaginaire négatif de César (le dictateur) pour rehausser la figure de La Fayette, celui-là recourt à l'imaginaire positif de César (le général mainte fois victorieux) pour rabaisser la figure de La Fayette.

Dans le discours révolutionnaire, l'imagerie romaine ne vaut donc pas pour elle-même : elle s'inscrit dans une perspective polémique, et son rejet s'accompagne presque toujours d'un ensemble de reprises et de variations qu'il importe de prendre en compte pour véritablement en saisir la portée. C'est à travers ce perpétuel dialogue que se construit un réseau de figures, qui accroît leur efficacité et qui diffuse un imaginaire romain issu de l'Ancien Régime dont les significations changent, puisqu'il est utilisé pour mettre des mots sur une civilisation naissante: les figures de la rhétorique classique, elles aussi, font leur révolution. 


\section{Conclusion}

Bien loin d'être des formes figées tournées vers le passé, les figures du discours révolutionnaire - incluses dans l'usage généralisé d'une rhétorique inspirée du modèle cicéronien - doivent être analysées dans le contexte polémique de leur énonciation. La méfiance générale à l'encontre des ressources de la rhétorique, et le refus de la figure au nom de la raison et de la vérité, lieux communs hérités des Lumières, deviennent aussi incontournables, dans le discours des orateurs de la Révolution française, que les figures elles-mêmes, souvent issues d'un imaginaire romain indispensable à l'efficacité du discours, dans la mesure où c'est sa dimension sensible, notamment visuelle, qui met en branle les émotions nécessaires à l'émergence d'un ordre nouveau et à la consolidation d'une communauté nationale.

Cet imaginaire, qui nous semble un cliché, à nous qui contemplons la tempête depuis notre lointain rivage et qui sommes marqués par l'héritage d'un discours thermidorien critique à l'encontre de l'imagerie romaine et construit sur un ethos prétendument antirhétorique, est en fait en perpétuelle métamorphose dans les discours de toute la période. Alors que les institutions religieuses et scolaires d'Ancien Régime avait, du point de vue révolutionnaire, perverti le sens de l'imaginaire romain en l'adaptant au contexte chrétien et monarchique, les orateurs de la Révolution cherchent à en restaurer le sens véritable, à le régénérer selon un mot qu'ils affectionnent. Ainsi, il retrouve un sens et recommence à désigner le réel, voire à en dévoiler le sens, avant de se figer à nouveau dans les discours épidictiques à la gloire de l'Empereur à la fin de la période.

\section{NOTES}

1. L.-S. Mercier, Tableau de Paris, nouvelle édition corrigée et augmentée, t. III, Amsterdam, [s.n.], 1782, p. 150.

2. H. Taine, Les origines de la France contemporaine, t. III (« La Révolution : la conquête jacobine »), Paris, Éditions Robert Laffont, « Bouquins », 1986, p. 269.

3. G. Lanson, Histoire littéraire de la France, Paris, Hachette, 1896, p. 850-851.

4. En réalité, au sein de notre corpus, exemple et exemplum se confondent souvent, l'auditeur devant généralement déduire à la fois un lien entre passé romain et actualité politique et tirer de l'exemple une règle morale universelle.

5. Ce genre de parallèle représente, dans le discours, l'équivalent des parallèles littéraires imités des Vie parallèles des hommes illustres de Plutarque, très à la mode au tournant des Lumières.

6. À titre d'exemple, on peut mentionner le discours que prononce Louvet le 29 octobre 1792, à l'occasion du procès du roi. Orateur girondin, Louvet souhaite faire exiler Philippe d'Orléans (député du camp adverse - montagnard - et lié à la famille royale). Il substitue alors la voix et les paroles de Brutus (d'après l'Histoire romaine de Tite-Live) aux siennes propres, et « jure que c'est Brutus qui parle » et qu'il n'en est « que

Exercices de rhétorique, 15 | 2020 
l'interprète fidèle » (J.-B. Louvet, discours prononcé le 16 décembre 1792 à la Convention, Gazette nationale ou Le Moniteur universel, 1792, $\mathrm{n}^{\circ}$ 353, Réimpression de l'ancien Moniteur depuis la réunion des états-généraux jusqu'au Consulat, Paris, 1840-1845, t. 14, p. 763). L'orateur acquiert alors le pouvoir de faire parler les morts, lesquels, dans ce qu'on pourrait presque qualifier de nekuia, semblent se substituer purement et simplement à l'orateur.

7. On retrouve cette expression de P. Ricœur aussi bien dans La Métaphore vive que dans Temps et récit (où le philosophe approfondit les thèses défendues dans les deux dernières études de La Métaphore vive). Selon Ricœur, la métaphore, en substituant à un sens littéral un sens figuré, invente le réel, au sens où elle le dévoile, à la manière dont le mythos dévoile une vérité cachée en arrachant le cours des événements à la contingence. Il s'agit donc d'une « logique de la découverte » (P. Ricœur, La Métaphore vive, première étude « Entre rhétorique et poétique : Aristote ", Paris, Éditions du Seuil, «Points essais », 1975, p. 18).

8. Quintilien, Institution oratoire, VIII, 3, 61-62, trad. J. Cousin, Paris, Les Belles-Lettres, 1978, t. V, p. 77-78.

9. Pour Aristote comme pour Cicéron, les expressions figurées, et tout particulièrement les métaphores, ont pour fonction principale de mettre l'objet sous les yeux de l'auditeur. Aristote explique que, dans la métaphore, « ce qui a du succès, c'est [...] de mettre la chose sous les yeux » (Aristote, Rhétorique, III, 10, 1410b34, trad. P. Chiron, Paris, Flammarion, 2007, p. 472). Cicéron ajoute que « les métaphores [...] s'adressent directement à nos sens, et particulièrement aux yeux, le plus pénétrant des sens ", ce qui leur permet de « [rendre] pour ainsi dire visible à l'esprit ce que nous ne pouvons distinguer et voir » (Cicéron, De l'orateur, II, 94, trad. E. Courbaud, Paris, Les BellesLettres, 2009, p. 63).

10. L.-A. de Saint-Just, Institutions républicaines, Euvres complètes, Paris, Gallimard, 2004, p. 1101.

11. A. Quennedey, « Le talent oratoire est-il un danger pour la liberté ? La controverse sur l'éloquence dans le discours de Saint-Just du 9 thermidor an II ", dans C. Triolaire dir., La Révolution française au miroir des recherches actuelles, actes du colloque tenu à Ivrysur-Seine les 15 et 16 juin 2010, Paris, Société des études robespierristes, 2011, p. 195. 12. L.-A. de Saint-Just, discours du 9 thermidor an II, Euvres complètes, op. cit., p. 770 (nous soulignons). En réalité, Saint-Just n'a pas le temps de prononcer ce discours : il est interrompu au bout de quelques phrases par les thermidoriens.

13. Comme tous les autres groupes ou « camps " politiques de la Révolution française, les constitutionnels ne forment pas un parti bien défini, mais plutôt une tendance. On désigne par ce terme, dans l'assemblée constituante puis législative, les partisans d'une monarchie constitutionnelle qui cherchent à conserver certains pouvoirs à l'exécutif, c'est-à-dire au roi.

14. Les 31 mai et 2 juin 1793 sont deux journées insurrectionnelles qui opposent la Convention à la Commune et aux assemblées de section. À l'issue de ces deux journées, de nombreux orateurs girondins sont arrêtés.

15. La commission extraordinaire des Douze (composée essentiellement de députés rattachés à la Gironde) est chargée de veiller à la tranquillité intérieure de la république. En réalité, elle surveille essentiellement les activités de la Commune, alors plutôt soutenue par les montagnards.

16. D. J. Garat, Mémoire sur la Révolution, ou exposé de ma conduite dans les affaires et dans les fonctions publiques, Paris, J. J. Smits, 1795, p. 112. 
17. Ibid., p. 85-86. En l'occurrence, Garat juge abusive l'analogie sans cesse brandie par les girondins entre leur situation et la conjuration de Catilina. Il souhaiterait plutôt les voir suivre la manœuvre de Cicéron lorsqu'il se ménagea hypocritement les faveurs de son collègue et adversaire, le consul Caius Antonia Hybrida, afin d'éviter que celui-ci ne se rallie à Catilina. Mais cet épisode, autrement moins héroïque que celui de la conjuration tel qu'il est éloquemment déployé dans les Catilinaires, n'est évidemment pas prégnant dans l'imaginaire collectif.

18. Ibid., p. 52.

19. Ibid., p. 82.

20. M. J. Lequinio, Les préjugés détruits, Paris, Imprimerie Nationale (chez Desenne et Debray), 1792, p. 34.

21. Ibid., p. 41.

22. J. v. Dumolard, discours prononcé le 21 juillet 1792 à l'assemblée législative, Gazette nationale ou Le Moniteur universel, 1792, $\mathrm{n}^{\circ}$ 205, Réimpression de l'ancien Moniteur, t. 13, p. 208.

23. J. Pétion, Gazette nationale ou Le Moniteur universel, 1792, $\mathrm{n}^{\circ} 315$, Réimpression de l'ancien Moniteur, op. cit., t. 14, p. 427.

24. Ibid., p. 430.

25. Ibid., p. 428.

26. É. Guadet, discours prononcé le 12 avril 1793 à la Convention, Gazette nationale ou Le Moniteur universel, 1793, n 105, Réimpression de l'ancien Moniteur, op. cit., t. 16, p. 133.

27. B. Barère, discours prononcé le $1^{\mathrm{er}}$ août 1793 à la Convention, Gazette nationale ou Le Moniteur universel, 1793, n 219, Réimpression de l'ancien Moniteur, op. cit., t. 17, p. 327. 28. M. Robespierre, discours prononcé le 26 juillet 1794 ( 8 thermidor an II) à la Convention, Euvres de Maximilien Robespierre, Discours ( $5^{e}$ partie), 27 juillet $1793-27$ juillet 1794, t. X, Paris, Société des études robespierristes, 2011, p. 568.

29. Ibid., p. 565.

30. Le 28 juin, le général La Fayette, quittant son poste à la tête de son armée, vient adresser une pétition à la barre de l'assemblée législative. Il demande la fermeture du club des Jacobins, qu'il accuse de fomenter des troubles intérieurs. Cette pétition marque le début de l'affaire La Fayette : elle provoque un tollé chez les députés jacobins, qui accusent le général d'aspirer à la dictature, tandis que les constitutionnels prennent sa défense.

31. J. v. Dumolard, op. cit., p. 209.

32. É. Guadet, op. cit., no 104, p. 127.

33. M. Robespierre, op. cit., p. 567.

34. J.-B. Louvet, discours prononcé le 29 octobre 1792 à l'assemblée législative, Gazette nationale ou Le Moniteur universel, 1792, $\mathrm{n}^{\circ}$ 305, Réimpression de l'ancien Moniteur, op. cit., t. 14, p. 342. - « Masaniel » est Masaniello (1620-1647), révolutionnaire napolitain qui s'insurgea contre la Couronne espagnole.

35. J.-B. Louvet, Archives parlementaires de 1787 à 1860, sous la dir. M.-J. Madival et M. E. Laurent, première série (1797 à 1799), t. 53, p. 53.

36. Ibid., p. 54.

37. Ibid.

38. M. Robespierre, discours prononcé le 5 novembre 1792 à l'assemblée législative, Gazette nationale ou Le Moniteur universel, 1792, n 311, Réimpression de l'ancien Moniteur, op. cit., t. 14, p. 390. 
39. Ibid., p. 392.

40. C.-F. Chassebœuf de la Giraudais, dit Volney, Leçons d'histoire à l'École normale en l'an III de la République française, éd. J. Gaulmier, Paris, Garnier, 1980, p. 140-141.

41. F.-J de La Harpe, De la guerre déclarée par les Tyrans révolutionnaires à la Raison, à la Morale, aux Lettres et aux Arts, Discours prononcé à l'ouverture du Lycée le 31 décembre 1794, Lycée ou cours de littérature ancienne et moderne, t. VIII, Paris, Depélafol, 1875, p. 9-10.

42. Ibid., p. 10. Les italiques, présents dans la version imprimée du discours de La Harpe ou ajoutés par l'éditeur, marquent les termes employés en autonymie, empruntés avec une ironie en mention acerbe au discours jacobin.

43. Ibid.

44. Le député Courtois présente, le 5 janvier 1795, un très long rapport judiciaire faisant état des documents trouvés chez Robespierre et ses « complices ». Or, ce rapport alterne curieusement entre une présentation objective de ces documents, parfois cités in extenso, et des commentaires abondants relevant de la grande éloquence déclamatoire. Les figures d'analogie romaines sont omniprésentes, parfois présentées sur le mode de la prétérition, comme dans ce passage : " Sans tomber dans la fureur des parallèles, je ne sais qu'un certain Domitius, dont parle Salluste, [...] offre quelques traits de ressemblance avec Robespierre [...] » (E.-B. Courtois, Rapport fait au nom de la commission chargée de l'examen des papiers trouvés chez Robespierre et ses complices, par E.-B. Courtois, député du département de l'Aube, dans la séance du 16 nivôse an III de la république française une et indivisible, 5 janvier 1795, Gazette nationale ou Le Moniteur universel, an III, $\mathrm{n}^{\circ} 151$, Réimpression de l'ancien Moniteur, op. cit., t. 23, p. 485).

45. L. M. S. Fréron, discours prononcé à la Convention le 27 juillet 1794 ( 9 thermidor an II), Gazette nationale ou Le Moniteur universel, an II, $\mathrm{n}^{\circ} 312$, Réimpression de l'ancien Moniteur, op. cit., t. 21, p. 341.

46. C'est le discours thermidorien qui a initié la vision négative de tout un courant historiographique anti-jacobin, lequel a largement servi de relais à l'idée que l'éloquence déclamatoire et figurée du discours révolutionnaire était l'apanage des Jacobins et contribuait à expliquer la violence de la « Terreur », idée qui circule encore largement aujourd'hui.

47. « Le discours polémique suppose, comme pour l'essai, un milieu topique sousjacent, c'est-à-dire un terrain commun entre les entreparleurs » (M. Angenot, La Parole pamphlétaire, typologie des discours modernes, Paris, Payot, «Langage et société », 1995, p. 35).

48. Voir supra, p. 4.

49. L.-A. de Saint-Just, op. cit., p. 780-781.

50. J. Droz, Essai sur l'art oratoire, chap. IX : « Des formes qui annoncent le travail de l'esprit, et de celles qui conviennent au sentiment. Observations sur les figures. », Paris, Renouard, 1800, p. 93-121.

51. Ibid., p. 93.

52. Ibid., p. 93-94.

53. Ibid., p. 96.

54. Ibid., p. 121.

55. J. V. Dumolard, op. cit., p. 208.

56. Ibid.

57. Ibid.

58. Voir supra, p. 7. 
59. V. M. Viénot de Vaublanc, discours prononcé le 8 août 1792 à l'assemblée législative, Gazette nationale ou Le Moniteur universel, 1792, n²22, Réimpression de l'ancien Moniteur, op. cit., t. 13, p. 359.

60. L'anté-occupation est une figure par laquelle l'orateur « feint qu'un tiers, ou l'adversaire, ou simplement l'interlocuteur, lui oppose une objection et l'exprime » (G. Molinié, Dictionnaire de rhétorique, Paris, Le livre de poche, 1992, p. 52). Ici, il ne s'agit pas à proprement parler d'une anté-occupation, car le locuteur ne mentionne pas une opinion de ses adversaires, mais sa propre opinion initiale : il aurait dû défendre La Fayette avec une « froide modération », mais les circonstances lui imposent de faire autrement (c'est aussi une manière de renforcer le caractère exceptionnel de la situation).

61. V. M. Viénot de Vaublanc, discours prononcé le 8 août 1792 à l'assemblée législative, Archives parlementaires de 1787 à 1860, op. cit., t. 47, p. 564.

62. Ibid.

63. Id., discours prononcé le 8 août 1792 à l'assemblée législative, Gazette nationale ou Le Moniteur universel, op. cit., p. 363.

64. J.-P. Brissot, discours prononcé le 8 août 1792 à l'assemblée législative, Archives parlementaires, op. cit., t. 47, p. 572.

65. Ibid.

66. J. v. Dumolard, op. cit., p. 208.

\section{AUTEUR}

\section{HÉLÈNE PARENT}

Université Paris Nanterre - Centre des sciences des littératures en langue française 